\title{
An Enhancement Neighborhood Connected Segmentation for 2D-Cellular Image
}

\author{
M. M. Abdelsamea
}

\begin{abstract}
A good segmentation result depends on a set of "correct" choice for the seeds. When the input images are noisy, the seeds may fall on atypical pixels that are not representative of the region statistics. This can lead to erroneous segmentation results. In this paper, an automatic seeded region growing algorithm is proposed for cellular image segmentation. First, the regions of interest (ROIs) extracted from the preprocessed image. Second, the initial seeds are automatically selected based on ROIs extracted from the image. Third, the most reprehensive seeds are selected using a machine learning algorithm. Finally, the cellular image is segmented into regions where each region corresponds to a seed. The aim of the proposed method is to automatically extract the Region of Interests (ROI) from the cellular images in terms of overcoming the explosion, under segmentation and over segmentation problems. Experimental results show that the proposed algorithm can improve the segmented image and the segmented results are less noisy as compared to some existing algorithms.
\end{abstract}

Index Terms-Image segmentation, Seeded region growing, Machine Learning, Leaking problem.

\section{INTRODUCTION}

Quantitative studies have been performed based on populations of biological images. Such studies extremely require methods for segmentation, feature extraction, and classification.

A first step in many analysis pipelines is segmentation, which can occur at several levels (e.g., separating nuclei, cells, tissues). This task has been an active field of research in image processing over the last 30 years, and various methods have been proposed and analysed depending on the modality, quality, and resolution of the microscopy images to analyze.

For image segmentation, there are four main approaches: threshold techniques, boundary based techniques, region based techniques, and hybrid techniques [1].

In threshold techniques [2] all pixels whose value (gray level, color value, or other) lie within a certain range can be classified as one class. Such methods neglect all of the spatial information of the image and do not cope well with.

Boundary based methods [3] use the postulate of the rapid changes of the pixel values at the boundary between two regions. The basic method here is to apply a gradient operator (e.g., Sobel, Roberts filter). High values of this filter provide candidates for region boundaries, which must then be modified to produce closed curves representing the

Manuscript received November 4, 2011; revised November 20, 2011.

M. M. Abdelsamea is with Mathematics Department, Faculty of Science, Assiut University, Assiut, Egypt (e-mail: mm_abdelsamea@yahoo.com). boundaries between regions. Converting the edge pixel candidates to boundaries of the regions of interest is a difficult task. The complement of the boundary-based approach is to work with the regions.

Region based methods [4] rely on the postulate that all neighbouring pixels within the one region have similar value or a specific range. This leads to the class of algorithms known as region growing of which the "split and merge" technique is probably the best known. The general procedure is to compare a specific feature of one pixel to its neighbor(s) feature. If a criterion of homogeneity is satisfied, the pixel is classified to the same class as one or more of its neighbors. The choice of the homogeneity criterion is critical for even moderate success and in all instances the results are upset by noise.

The fourth type is the hybrid techniques [5] which combine boundary and region criteria. This class includes morphological watershed segmentation and variable-order surface fitting. The watershed method is generally applied to the gradient of the image. This gradient image can be viewed as topography with boundaries between regions as ridges. Segmentation is equivalent to flooding the topography from the seed points with region boundaries being erected to keep water from different seed points from meeting. As same as all pervious method the technique encounters difficulties with images in which regions are both noisy and have blurred or indistinct boundaries. Furthermore, method is also computationally very expensive.

In this paper, an accurate segmentation method is proposed based on the seeded region growing method to be less sensitive to the noisy image and quantified and avoid explosion, leaking, under segmentation, and over segmentation problems.

\section{RELATED WORK}

Jie Wu et al [6] proposed a new texture feature-based seeded region growing algorithm for automated segmentation of organs in abdominal MR images. The proposed seed point determination method is based on a cost-minimization approach. An ideal candidate seed point should have these properties: i) It should be inside the region and near the center of the region ii) Assume most of the pixels in the ROI belong to the region (i.e. ROI is not too big compared to the region), the feature of this seed point should be close to the region average iii) The distances from the seed pixel to its neighbors should be small enough to allow continuous growing.

Mehnert and Jackway [7] pointed out that Seeded Region Growing (SRG) has two inherent pixel order dependencies 
that cause different resulting segments. The first-order dependency occurs whenever several pixels have the same difference measure to their neighboring regions. The second-order dependency occurs when one pixel has the same difference measure to several regions. They used parallel processing and re-examination to eliminate the order dependencies.

Frank et al [8] present an automatic seeded region growing algorithm for color image segmentation. In this paper, they apply the SRG to color images with automatic seed selection. They develop strategies to avoid the two order dependencies. For automatic seed selection, the following three criteria must be satisfied. First, the seed pixel must have high similarity to its neighbors. Second, for an expected region, at least one seed must be generated in order to produce this region. Third, seeds for different regions must be disconnected.

Whitney et al. [9] overcomes the need to manually select threshold values by analyzing the histogram of voxel similarity to automatically determine a stopping criterion, but they still require the user to choose a seed point.

Law et al. [10] proposed a Genetic Algorithm based seed selection method and a threshold value optimization method, but their algorithm has the problem of possible under-segmentation and speed can also be an issue although they did not address them.

There are a number of known issues associated with SRG scheme:
A good segmentation result depends on a set of "correct" choice for the seeds. When the input images are noisy, the seeds may fall on atypical pixels that are not representative of the region statistics. This can lead to erroneous segmentation results.

The seed selection process in itself requires manual interventions, and is error-prone. Even though automatic segmentation can be achieved in a limited sense, it is application specific and will require domain-specific knowledge and training sets.

All of these make SRG unsuitable for segmentation where a priori knowledge is limited. The main idea of the proposed algorithm is to overcoming these drawbacks.

\section{Methodology OvervieW}

The system consist of three stages: i) After the preprocessed image is loaded into the system, the ROIs extracted via Otsu image segmentation from the image as a binary object; ii) a proposed method is applied for automatically selected seeds based on positions and intensities of pixels located in ROIs extracted; iii) a region growing procedure is recursively called for segment an input image as a seeded region growing based on seeded candidates and Otsu threshold. Fig. 1 illustrates the overview of the proposed algorithm.

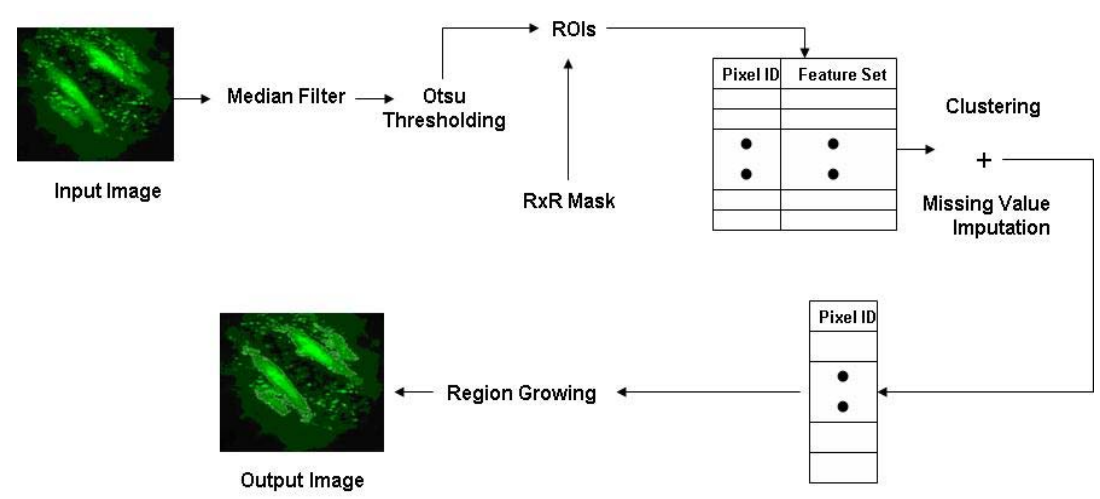

Fig. 1. Outline of the proposed algorithm.

\section{The Method For SeEd SElection}

Seeded region growing performs a segmentation of an image with respect to a set of points, known as seeds. We start with a number of seeds which have been grouped into $\mathrm{n}$ sets, say, A1, A2, ...,An. Given a seed point, the region growing method searches the seed point's neighbors to determine whether they belong to the same region. If they are determined to be so, their neighbors are searched. The process is recursively executed until no more new neighbors can be added to the region [11].

The criterion is when the distance is lower than a hreshold value a neighbor point is added. So we need to determine the distance measure, linkage strategy, connectivity strategy and a threshold value [12], See Fig.2.

For automatic seed selection, an ideal candidate must have the following properties and satisfy the following criteria: i) Make sure that the seed point candidate is not on the boundary of two regions and/or not outlier.

ii) Check whether this candidate seed point has high similarity to its neighbours, which it should be inside the selected ROI and has the highest similarity between its neighbors to accurately represent the region.

iii) At least one seed pixel must be generated for each extracted ROI.

iv) The distances measured from the seed pixel to its neighbors should be inside extracted region to allow continuous growing.

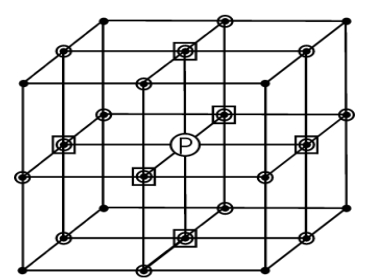

Fig. 2. 6-(squares), 18-(circles), and 26-neighbors (dots). 
For satisfy these criteria the proposed segmentation describes as follow:

The first segmentation step after image acquisition tries to reduce the noise present in the image. To reduce noise several non-linear filters can be employed. One of the simplest techniques, the median filter, can be employed here given that it provided good noise reduction without affecting the borders of the objects on the image.

Secondly, an Otsu segmentation method is applied in order to: extract ROIs from the input image and extract Otsu threshold, threshold that classifies the image into two clusters such that we minimize the area under the histogram for one cluster that lies on the other cluster's side of the threshold, to be used later as input in region growing procedure.

The aim of the filtering step is to remove outliers points and points located on the boundary of ROIs by considering and using R X R neighborhood mask to make sure that all the tested seed point's neighbors located within this mask, A particular pixel will be considered as a boundary pixel or outlier if at least one of all its neighbours is determined as a background pixel. The size of the neighbourhood to be considered around the pixel is determined by a radius value R.

Thirdly, candidate seeds positions and its intensity values are extracted as features to represent the training set, which in this case the candidate seeds are the patterns of the training set, and apply it for clustering via machine learning technique; in this work K-mean clustering technique has been used.

After training process, the training pattern is applied as a test pattern to the learned algorithm and $\mathrm{K}$ is specified accordingly, which $\mathrm{K}$ is number of seeds or number of object that user would like to extract, to get the most representive point to use as seeds for the Region Growing method for segment image into region where each region corresponds to a seed.

In Seed Extraction, we can consider extracting of the seed as a Missing Value Imputation problem which can be solved by extracting the prototypes of the positions of the centroids to act as an ideal seeds. By this step we make sure that this candidate seed has the highest similarity between its neighbors, at least one seed must be generated for each expected region, and the distances measured from the seed pixel to its neighbors should be inside extracted region to allow continuous growing.

It's obviously clear that this proposed method can avoid the explosion, under-segmentation and over-segmentation problems.

\section{How to quantify an explosion?}

By using the concept of considering the neighbors for growing the region, the object will not grow over its boundary.

\section{How to avoid under-segmentation?}

1. Using Otsu threshold with the main concepts of the growing region strategy have been used in this work, allow extract the whole region and make a stop before explosion.

2. make sure that the seed point candidate is not on the boundary of two regions.

\section{How to avoid over-segmentation?}

At least one seed pixel must be generated for each expected region, and Make sure that this candidate seed has the highest similarity between its neighbors to accurately represent the region.

How to determine whether a neighbor pixel belongs to the same region?

The criterion is when the distance is lower than a threshold value a neighbor point is added. So we need to determine the distance measure, linkage strategy, connectivity strategy and a threshold value.

Distance measure:

The Euclidean distance in the feature space is used as the distance measure. For example, the distance between two pixels is the Euclidean distance of their feature vectors.

\section{Linkage strategy:}

In single linkage, the pairs of neighboring pixels are compared for merging, is one of the conceptually simplest approaches.

In centroid linkage, a pixel's value is compared with the mean of an already existing but not necessarily completed region.

Single linkage strategy has been chosen over centroid linkage because it is faster and more memory efficient considering the calculation of texture features and recursively running $S R G$ requires much memory.

\section{Connectivity strategy:}

In $2 \mathrm{D}$ region growing, there are two connectivity strategies that people use: four-neighbor and eightneighbor. Four-neighbor region growing checks only the vertically and horizontally connected four neighbors, while eight-neighbor region growing checks vertically, horizontally and diagonally connected eight neighbors. The choice of neighbor connection strategy is usually case dependent.

An optimal threshold value is the value that can make a stop to the region growing and the obtained region is optimal. It is desirable that the threshold value is high enough to extract the whole region; however if the threshold value is higher than the optimal one, the extracted region may grow over the actual region boundary and grow to a much larger region. This case is called 'explosion'

\section{SeEded Region Growing}

For growing the region based on the extracted seed, it's desirable to use the same principle of the filtering which has been used in this work to avoid the explosion and under segmentation that might be happen because of the leaking problem of the region growing. For this reason a neighbourhood condition has been applied for accepting the pixel to be including in the region, these conditions based on the intensity value by considering an Otsu Threshold as an optimal threshold.

By the principle of neighbourhood connected, growing the region is not completely depend on the feature of interest determined by the seed, the feature of interest that determined by the seed is just a position. 


\section{EXPERIMENTAL RESULTS}

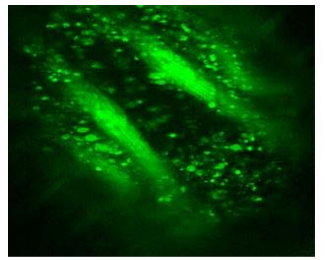

a1

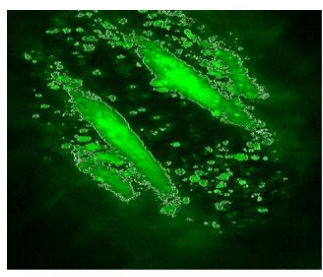

a3

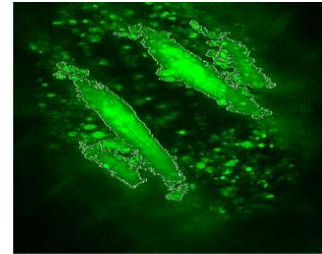

a2

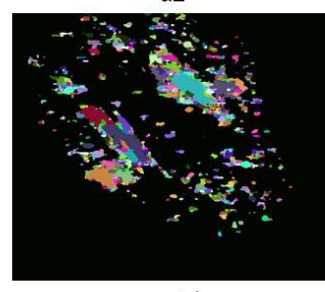

a4
Fig. 3. (a1) Original image, (a2) the result of segmentation using the proposed algorithm with $\mathrm{K}=4$ and $\mathrm{R}=3$, (a3) the segmentation result using Otsu method, (a4) the segmentation result using watershed method with $\mathrm{L}=.08$ and $\mathrm{T}=.08$.

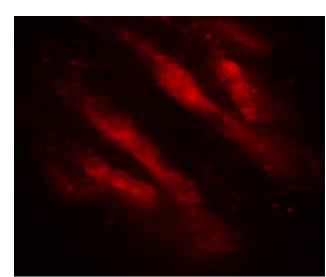

a1

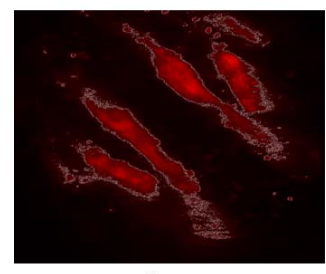

a3

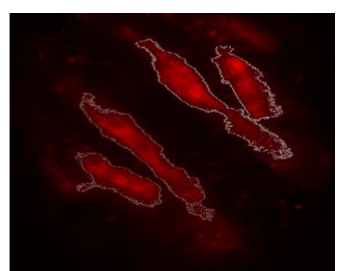

a2

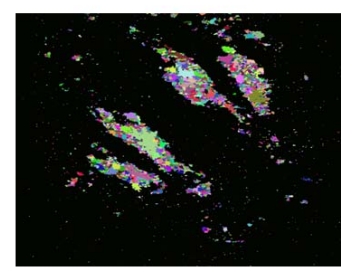

Fig. 4. (a1) Original image, (a2) the result of segmentation using the proposed algorithm with $\mathrm{K}=4$ and $\mathrm{R}=3$, (a3) the segmentation result using Otsu method, (a4) the segmentation result using watershed method with $\mathrm{L}$ $=.05$ and $\mathrm{T}=.05$.

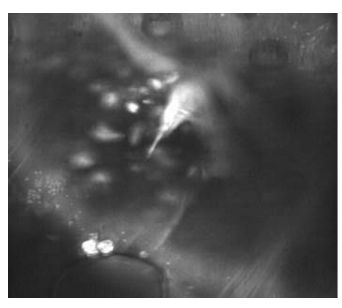

a1

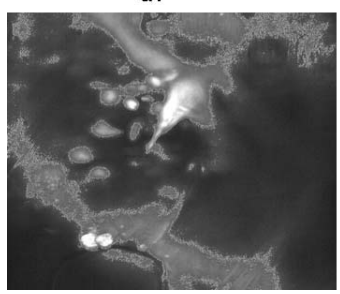

a3

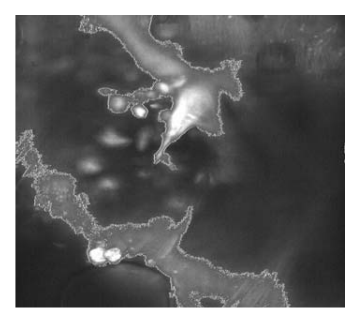

a2

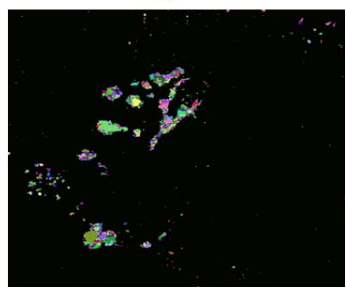

a4
Fig. 5. (al) Original image, (a2) the result of segmentation using the proposed algorithm with $\mathrm{K}=3$ and $\mathrm{R}=3$, (a3) the segmentation result using Otsu method, (a4) the segmentation result using watershed method with $\mathrm{L}=5$ and $\mathrm{T}=5$, (a5) the segmentation result using JSEG.

I have performed the experiments using the proposed segmentation algorithm and performed comparisons with some existing segmentation algorithm. In Fig 3, 4, 5, and 6, we show the comparison with Otsu algorithm, Watershed algorithm, and JSEG algorithm [13].

By observing Fig 3(a3, a4), Fig 4(a3, a4), and Fig 5(a3, a4) we can realize that their results are over segmented. Segmented objects in Fig 3(a5), Fig 4(a5), and Fig 6 are inappropriately merged to background.

By comparing these results with results of our proposed algorithm in Fig 3(a2), Fig 4(a2), and Fig 5(a2) we can clearly notice that the proposed segmentation algorithm overcoming the over-segmentation problem and is less noisy as compared to other algorithms.
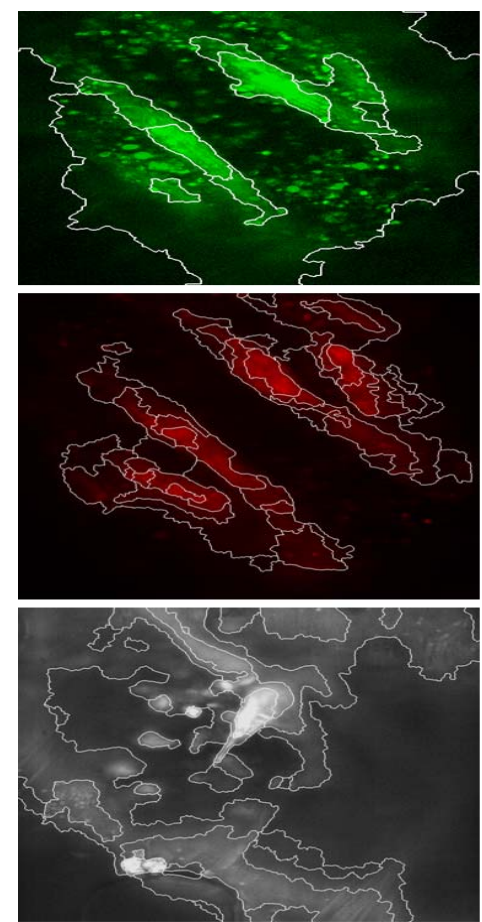

Fig. 6. The result of segmentation using the JSEG algorithm.

\section{CONCLUSION}

The issues of SRG Algorithm can be summarized as: The selection of the seeds determines what a feature of interest is and what is irrelevant. So, growing the region is completely depending on the feature of interest determined by the seed.

In this paper, an automatic seeded region growing framework for automatically segment $2 \mathrm{D}$ cellular image is proposed. One of the advantages of this algorithm is obvious, as it provides a parameter free production environment to allow minimum user intervention. This can be especially helpful for batch work or to novice computer users. The other benefit of this algorithm is overcoming the explosion, under segmentation and over segmentation problems.

Furthermore, it is clearly that the proposed algorithm able to consuming time because only specific regions are used to produce seeds.

However, the selection of $\mathrm{k}$, number of expected regions might lead to miss-segmentation. Check whether this candidate seed has the highest similarity between its neighbors is sensitivity to the selection of the number of the expected regions, which might lead to miss-segmentation.

The issue can be summarized as: The sensitivity to how to expect the region and the number of expected regions 
might lead to extract seed inaccurately represent the region.

The future work includes investigation of other features like texture features as a feature for selecting seeds and growing the region.

\section{ACKNOWLEDGMENT}

I would like to thank Imaging Informatics group, Bioinformatics Institution - Singapore, to provide the test images that have been used in this work and for the useful discussions and constructive comments.

\section{REFERENCES}

[1] P. J. Besl and R. C. Jain, "Segmentation through variable-order surface fitting," IEEE Trans. Pattern Anal. Machine Intell., vol. PAMI-IO, pp. 167-192, 1988.

[2] P. K. Sahoo, S. Soltani, A. K.C. Wong, and Y. C. Chen. 1988. A survey of thresholding techniques. Comput. Vision Graph. Image Process. 41, 2 (February 1988), 233-260.

[3] L. S. Davis, "A survey of edge detection techniques," Comput. Graph. Image Process., vol. 4, pp. 248-270, 1975.

[4] S. W. Zucker, "Region growing: Childhood and adolescence," Comput. Graph. Image Process., vol. 5, pp. 382-399, 1976.s

[5] F. Meyer and S. Beucher, "Morphological segmentation," J. Vis. Commun. Image Represent. vol. 1, pp. 2146, 1990

[6] J. Wu, S. Poehlman, M. D. Noseworthy, M. Kamath, (2008) Texture Feature based Automated Seeded Region Growing in Abdominal MRI Segmentation, 2008 International Conference on Biomedical Engineering and Informatics, Sanya, China, May 27-30.

[7] A. Mehnert, P. Jackway, An improved seeded region growing algorithm, Pattern Recognition Letters 18 (1997) 1065-1071.
[8] FY Shih and S. Cheng, "Automatic seeded region growing for color image segmentation", Image and Vision Computing, Vol. 23, 2005, pp. $877-886$

[9] R. Adams and L. Bischof. Seeded region growing. IEEE Trans. Pattern Anal. Mach. ntelligence, 16(6):641-647, 1994.

[10] B. W. Whitney, N. J. Backman, J. D. Furst, D. S. Raicu, (2006) "Single click volumetric segmentation of abdominal organs in Computed Tomography images", Proceedings of SPIE Medical Imaging Conference, San Diego, CA, Februar.

[11] T.-Y. Law and P. A. Heng, (2000) "Automated extraction of bronchus from 3-D CT images of lung based on genetic algorithm and 3-D region growing”, Proc. SPIE 3979, Medical Imaging 2000: Image Processing, 906-916.

[12] Jie Wu, Skip Poehlman, Michael D. Noseworthy, Markad V. Kamath, "Texture Feature-based Automated Seeded Region Growing in Abdominal MRI Segmentation", Journal of Biomedical Science and Engineering, (Feb. 2009), pp.1-8.

[13] Y.Deng, and B.S.Manjunath, "Unsupervised segmentation of colortexture regions in images and video," IEEE Transactions on Pattern Analysis and Machine Intelligence (PAMI '01), August 2001.

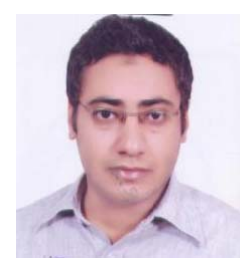

M. M. Abdelsamea, (M.Sc), he is born in Assiut, Egypt. He has received his Master degree in Computer Science "Medical Image Analysis" from Assiut University. He published and presented a number of peer-review papers in international conferences related to Medical Image Analysis. He worked as a member in a number of groups in Egypt and Singapore. He is a member of IACSIT and APCBEES. Currently, Abdelsamea works as an Assistant Lecturer at Mathematics Department, Faculty of Science in Assiut University. He is interested in Computer Vision, Machine Learning, and Image Segmentation. 